\title{
Use of adjuvant EGFR tyrosine kinase inhibitors in early stage EGFR-mutant non-small cell lung cancer: is the evidence strong enough?
}

\author{
Christopher Lemmon, Nathan A. Pennell \\ Department of Hematology and Medical Oncology, Cleveland Clinic Taussig Cancer Center, Cleveland, Ohio, USA \\ Correspondence to: Nathan A. Pennell, MD, PhD, FASCO. Cleveland Clinic Taussig Cancer Center, 9500 Euclid Avenue., CA-62, Cleveland, OH \\ 44195, USA. Email: penneln@ccf.org. \\ Provenance and Peer Review: This article was commissioned by the editorial office, Fournal of Thoracic Disease. The article did not undergo external peer \\ review. \\ Comment on: Liang W, Cai K, Chen C, et al. Society of Translational Medicine consensus on postoperative management of EGFR-mutant lung \\ cancer (2019 edition). Transl Lung Cancer Res 2019;8:1163-73.
}

Submitted Apr 30, 2020. Accepted for publication May 13, 2020.

doi: 10.21037/jtd-20-1817

View this article at: http://dx.doi.org/10.21037/jtd-20-1817

Targeted therapies have transformed the treatment of advanced non-squamous non-small cell lung cancer (NSCLC) when there are actionable driver mutations and are a consensus part of multinational guidelines (1-3). Activating epidermal growth factor receptor (EGFR) mutations and the tyrosine kinase inhibitors (TKI) that target them have been a premier example of this with improvements in response rates and progression free survival compared to traditional chemotherapy in the advanced setting (4). It is now standard practice to test for these mutations prior to initiating treatment to guide appropriate therapy, as well as to further understand the biology of the cancer at the time of disease progression and acquired resistance (5).

Naturally, with the success seen in the advanced setting and as targeted treatments have improved, the question has been raised if their use can be expanded to earlier stage disease with similar benefits, and with the added potential advantage of limiting the toxic effects of traditional chemotherapy. Several studies have attempted to explore this question but have not yet given a definitive answer. Comparisons between studies and broad applicability of their results is also difficult given the wide variability in populations and study designs.

An early study, SWOG S0023 looked at use of gefitinib as maintenance therapy in inoperable stage III patients after chemoradiation and docetaxel consolidation and found no benefit in disease free progression or survival, however this was an unselected population and did not specify EGFR sensitizing mutation status (6). Another study of unselected patients, BR19, looked at operable early stage patients given adjuvant gefitinib that again showed no benefit, and the subgroup of $E G F R$-mutant patients totaled only 7, limiting any strong conclusions (7).

A retrospective analysis did provide some insight in looking at 167 patients with EGFR-mutant NSCLC, 56 of whom received adjuvant EGFR TKI after chemotherapy, showing that those that received TKI had an improved disease-free survival (DFS) compared to those that did not (2-year DFS $89 \%$ vs. $72 \%$, respectively) (8). This early signal gave sufficient incentive to plan prospective trials.

The RADIANT trial was a large randomized, placebocontrolled phase III trial of adjuvant erlotinib for 2 years after surgery, but the patient population enrolled harbored EGFR protein or gene-copy overexpression and was not selected for activating $E G F R$ mutations. The trial did not show any evidence of clinical benefit in the overall population. The subgroup analysis of the EGFR-mutant NSCLC patients did show an improvement in DFS in the erlotinib group versus the placebo group (46.4 months vs. 28.5 months, respectively, HR $0.61,95 \%$ CI: $0.38-0.98$; $\mathrm{P}=0.39$ ) with a 2 -year DFS of $89 \%$ vs. $72 \%$ in the placebo group, but the study was not designed to look at the significance of this comparison if the overall population did 
not benefit, and this

subgroup had imbalances in population characteristics that limit the ability to draw conclusions (9). These results were generally consistent however with the prior retrospective analysis (8) and additional studies done specifically in EGFR-mutant populations, namely SELECT in the United States, and EVAN and ADJUVANT (CTONG1104) in China.

SELECT was a single arm phase 2 study of 100 EGFRmutation positive patients given 2 years of adjuvant erlotinib after finishing standard treatment for their stage IA to IIIA NSCLC, and provided further evidence supporting the notion that adjuvant TKIs improve DFS. The trial population had a 2-year DFS (88\%) and 5-year DFS (56\%) longer than historical controls, and also importantly provided information regarding relapse after termination of therapy, with only 4 out of 40 recurrences occurring while on therapy, suggesting a suppressive effect of treatment. It was not at all clear, however, that overall recurrences were reduced and not merely delayed, with an average time to recurrence after discontinuing therapy of 25 months. Additionally, there was shown to be minimal risk of developing acquired resistance with adjuvant treatment as only one patient out of those that recurred and were re-biopsied harbored the T790M resistance mutation (10).

The EVAN trial compared 2 years of erlotinib to standard platinum chemotherapy in a more limited patient population consisting of 102 Chinese stage IIIA EGFR-mutant NSCLC patients that had recently undergone resection. This again showed improvements in 2-year DFS (81.4\% vs. $44.6 \%$ in the chemotherapy group) and maintained 3-year DFS benefit of $54.2 \%$ vs. $34.4 \%$, respectively (11).

The largest adjuvant TKI trial in a mutant population was the ADJUVANT (CTONG1104) study, which used a similar construct of 2 years of gefitinib versus chemotherapy after surgery in recently resected stage II or IIIA EGFR-mutant NSCLC, though still limited to Chinese only centers. The study showed improvement in median DFS (28.7 months with gefitinib vs. 18 months with chemotherapy, HR 0.6, 95\% CI: $0.42-0.87 ; \mathrm{P}=0.005$ ) but by 3 years, the benefit seen was insignificant (3-year DFS 34\% vs. 27\%; HR 0.74, 95\% CI: $0.42-1.32 ; \mathrm{P}=0.37)$. There was also a high rate of relapse in their patient population treated with EGFR-TKI that was not seen in other studies (52\% as compared to $40 \%$ in SELECT and 35\% in EVAN) (12). This may be due to the inclusion of a larger percentage of stage IIIA patients in ADJUVANT, and also patients were enrolled immediately after resection, potentially including those with early recurrence that were not included in other studies where enrollment occurred after a short interval time.

All of the studies reported to-date have used firstgeneration EGFR TKIs like gefitinib and erlotinib, but the current standard of care in advanced EGFR-mutant NSCLC is the third-generation TKI osimertinib, established by improved overall survival (OS) head-to-head with older TKIs (13). Based on this, the ongoing phase 3 ADAURA trial (NCT02511106) was planned, with DFS as a primary endpoint. This trial is comparing 3 years of osimertinib versus placebo in the adjuvant setting for resected $E G F R$ mutant stage IB-IIIA NSCLC patients, and a recent press release announced that on interim analysis this trial had been stopped by the Data and Safety Monitoring Committee for "overwhelming efficacy" in the osimertinib arm $(14,15)$. Although an improvement is DFS would not be surprising given prior trials in this space, it remains to be seen if the magnitude of DFS will be enough to change treatment if OS data is not yet available.

While the studies above show that adjuvant EGFR TKIs consistently prolong DFS, importantly, no trial has shown a benefit in overall survival to this point. Overall survival has long been the standard for showing benefit and is the most important endpoint when studying a disease where the goal of treatment is cure. DFS is an appealing surrogate endpoint because differences can be detected much earlier than with OS, but it isn't clear in NSCLC that there is direct correlation between DFS and OS, especially when a highly effective therapy such as osimertinib is available at recurrence. The small benefit seen with chemotherapy in the adjuvant setting in early stage NSCLC is thought to derive from the cytotoxic destruction of microscopic disease and micrometastases (16). While EGFR-directed therapies are cytotoxic to a proportion of cells, they remain cytostatic to others, and this may lead to progression once these treatments are stopped, which is consistent with data from the studies above. The much-anticipated phase 3 ALCHEMIST trial (NCT02193282) looking at 2 years of erlotinib versus placebo after resection and standard treatment in EGFR-mutant NSCLC may help to give further clarity into the question of overall survival benefit, but results are not expected for some time (17).

Another issue with adjuvant TKIs is their possible impact on resistance to treatment at recurrence in those who are not cured. Importantly, the SELECT trial noted that many of the patients who progressed after discontinuation were re-challenged with the same TKI and appeared to have durable responses (10). The implications of this will require 
further investigation for confirmation, but suggests that patients treated with adjuvant TKI were not harmed even if not cured, and show promise in the ability to manage the disease at progression. There is also a question about the optimal duration of treatment given the potential for durable suppression of recurrence, but the potential for additive toxicities remains a mitigating factor. ADAURA may give some insight to this with its longer duration of treatment (3 years) and with the improved toxicity profile of osimertinib.

The recent guidelines put forth by the panel of authors from the Society of Translational Medicine attempt to provide direction and consensus to many of the questions related to managing EGFR-mutant patients after resection based on the data mentioned above (18). Their consensus statements identify the clear importance of the initial imaging evaluation, and of mutation testing at the time of diagnosis and recurrence that are aligned with recommendations from other guideline organizations (1-3). The issue of the addition of bone scans and brain MRI to routine surveillance chest imaging is not as clear. While there is evidence that the EGFR-mutated population is at increased risk for brain metastases (19), data supporting the utility of routine surveillance imaging are currently lacking.

The panel's most consequential recommendation concerns a support statement for the use of EGFR-TKI therapy for a duration of at least 2 years, either alone or in combination with chemotherapy, for $E G R F$-mutated patients after resection based on the DFS benefits reported in the previously discussed studies. However, given the lack of clear impact on long term recurrences and overall survival, broad recommendations supporting adjuvant TKI therapy cannot be supported as of yet, and their routine use in the adjuvant setting should be limited to use in a clinical trial. The DFS benefits are encouraging, but may not be meaningful in a disease group where there is potential for eradication and cure and where highly effective treatments are available for recurrence. There is some debate about their use in the adjuvant setting for those that are unable to tolerate chemotherapy, however dedicated study in this patient population has not occurred yet. Ultimately, these are the kinds of questions without definitive answers that occur frequently in real-life practice.

Would longer duration of TKI treatment, perhaps even indefinite treatment as is done in advanced disease, yield a long enough DFS to improve cure rates? ADAURA may help to answer this question, as well as the necessary question of what level of DFS benefit translates to improved
OS. Can toxicities be managed over long periods of treatment and how does this impact quality of life, especially important given that many surgically cured patients may be exposed to long periods of unnecessary treatment? How do patients fare with re-treatment of the same TKI after relapse? Ultimately, there are not currently enough data to provide clarity on these questions. Some studies in progress, ADAURA and ALCHEMIST, may provide some important insight especially in regards to duration, toxicity, and survival. There are also studies looking at EGFR-TKI use in the neoadjuvant setting that may shed some light on this field of investigation (NCT03433469, NCT04351555).

For now, adjuvant platinum-based chemotherapy alone remains the standard of care, even in the EGFR-mutant population, solely due to the small improvement in overall survival and the ability to provide cure in that subset of patients. Whether EGFR TKIs will change this scenario in the near future depends on ongoing trials showing an improvement in OS and ultimately cures.

\section{Acknowledgments}

Funding: None.

\section{Footnote}

Conflicts of Interest: Both authors have completed the ICMJE uniform disclosure form (available at http://dx.doi. org/10.21037/jtd-20-1817). NAP reports personal fees from Merck, personal fees from Eli Lilly, personal fees from BMS, personal fees from Amgen, personal fees from Astrazeneca, personal fees from Genentech, outside the submitted work. The other author has no conflicts of interest to declare.

Ethical Statement: The authors are accountable for all aspects of the work in ensuring that questions related to the accuracy or integrity of any part of the work are appropriately investigated and resolved.

Open Access Statement: This is an Open Access article distributed in accordance with the Creative Commons Attribution-NonCommercial-NoDerivs 4.0 International License (CC BY-NC-ND 4.0), which permits the noncommercial replication and distribution of the article with the strict proviso that no changes or edits are made and the original work is properly cited (including links to both the formal publication through the relevant DOI and the license). 
See: https://creativecommons.org/licenses/by-nc-nd/4.0/.

\section{References}

1. NCCN Clinical Practice Guidelines in Oncology: Nonsmall cell lung cancer. Version 3.2020. Available online: https://www.nccn.org

2. Postmus PE, Kerr KM, Oudkerk M, et al. Early and locally advanced non-small-cell lung cancer (NSCLC): ESMO Clinical Practice Guidelines for diagnosis, treatment and follow-up. Ann Oncol 2017;28:iv1-21.

3. Park K, Vansteenkiste J, Lee KH, et al. Pan-Asian adapted ESMO Clinical Practice Guidelines for the management of patients with locally-advanced unresectable non-smallcell lung cancer: a KSMO-ESMO initiative endorsed by CSCO, ISMPO, JSMO, MOS, SSO and TOS. Ann Oncol 2020;31:191-201.

4. Rosell R, Carcereny E, Gervais R, et al. Erlotinib versus standard chemotherapy as first-line treatment for European patients with advanced EGFR mutation-positive non-small cell lung cancer (EURTAC): A multicenter, open-label, randomized, phase 3 study. Lancet Oncol 2012;13:239-46.

5. Kalemkerian GP, Narula N, Kennedy EB, et al. Molecular Testing Guideline for the Selection of Patients With Lung Cancer for Treatment With Targeted Tyrosine Kinase Inhibitors: American Society of Clinical Oncology Endorsement of the College of American Pathologists/ International Association for the Study of Lung Cancer/ Association for Molecular Pathology Clinical Practice Guideline Update. J Clin Oncol 2018;36:911-9.

6. Kelly K, Chansky K, Gaspar LE, et al. Phase III trial of maintenance gefitinib or placebo after concurrent chemoradiotherapy and docetaxel consolidation in inoperable stage III non-small cell lung cancer: SWOG S0023. J Clin Oncol 2008;26:2450-6.

7. Goss GD, O'Callaghan C, Lorimer I, et al. Gefitinib versus placebo in completely resected non-small cell lung cancer: results of the NCIC CTG BR19 study. J Clin Oncol 2013;31:3320-6.

8. Janjigian YY, Park BJ, Zakowski MF, et al. Impact on disease-free survival of adjuvant erlotinib or gefitinib in patients with resected lung adenocarcinomas that harbor EGFR mutations. J Thorac Oncol 2011;6:569-75.

9. Kelly K, Altorki NK, Eberhardt WE, et al. Adjuvant erlotinib versus placebo in patients with stage IB-IIIA nonsmall cell lung cancer (RADIANT): a randomized, doubleblind, phase III trial. J Clin Oncol 2015;33:4007-14.

10. Pennell NA, Neal JW, Chaft JE, et al. SELECT: A phase
II trial of adjuvant erlotinib in patients with resected epidermal growth factor receptor-mutant non-small cell lung cancer. J Clin Oncol 2019;37:97-104.

11. Yue D, Xu S, Wang Q, et al. Erlotinib versus vinorelbine plus cisplatin as adjuvant therapy in Chinese patients with stage IIIA EGFR-mutation-positive non-small cell lung cancer (EVAN): a randomized, open-label, phase 2 trial. Lancet Respir Med 2018;6:863-73.

12. Zhong WZ, Wang Q, Mao WM, et al. Gefitinib versus vinorelbine plus cisplatin as adjuvant treatment for stage II-IIIA (N1-N2) EGFR-mutant NSCLC (ADJUVANT/ CTONG1104): a randomized, open-label, phase 3 study. Lancet Oncol 2018;19:139-48.

13. Soria JC, Ohe Y, Vansteenkiste J, et al. Osimertinib in untreated EGFR-mutated advanced non-small cell lung cancer. N Engl J Med 2018;378:113-25.

14. Wu YL, Herbst RS, Mann H, et al. ADAURA: Phase III, double-blind, randomized study of osimertinib versus placebo in EGFR mutation-positive early-stage NSCLC after complete surgical resection. Clin Lung Cancer 2018;19:e533-6.

15. ADAURA Press Release. Available online: https:// www.astrazeneca-us.com/content/az-us/media/pressreleases/2020/tagrisso-phase-iii-adaura-trial-will-beunblinded-early-after-overwhelming-efficacy-in-theadjuvant-treatment-of-patients-with-egfr-mutatedlung-cancer-04102020.html

16. Pignon JP, Tribodet H, Scagliotti GV, et al. Lung adjuvant cisplatin evaluation: a pooled analysis by the LACE Collaborative Group. J Clin Oncol 2008;26:3552-9.

17. Govindan R, Mandrekar SJ, Gerber DE, et al. ALCHEMIST trials: a golden opportunity to transform outcomes in early stage non-small cell lung cancer. Clin Cancer Res 2015;21:5439-44.

18. Liang W, Cai K, Chen C, et al. Society of Translational Medicine consensus on postoperative management of EGFR-mutant lung cancer (2019 edition). Transl Lung Cancer Res 2019;8:1163-73.

19. Li L, Luo S, Lin H, et al. Correlation between EGFR mutation status and the incidence of brain metastases in patients with non-small cell lung cancer. J Thorac Dis 2017;9:2510-20.

Cite this article as: Lemmon C, Pennell NA. Use of adjuvant EGFR tyrosine kinase inhibitors in early stage EGFR-mutant non-small cell lung cancer: is the evidence strong enough? J Thorac Dis 2020;12(9):5042-5045. doi: 10.21037/jtd-20-1817 